\title{
Administração à distância de drogas injetáveis em animais de médio e grande porte mediante o uso de besta*
}

\author{
Crossbow for ranged drug injection in large and medium sized animals \\ Fernando Lencastre Sicuro
}

\begin{abstract}
RESUMO
A administração de drogas intramusculares à distância em animais de zoológico, de criações extensivas e da fauna silvestre, envolve o uso de armas de ar comprimido ou de fogo, ambas de alto custo, ou então, dardos improvisados lançados por zarabatanas. Estes últimos apresentam resultados que ficam aquém do satisfatório em termos de distância e do volume de fármaco transportado. Tendo em vista a realidade econômica das instituições brasileiras de pesquisa veterinária e zoológica, foi desenvolvida uma flecha injetora com materiais de baixo custo, porém duradouros, para ser usada em bestas comerciais ou de fabricação artesanal, com grande eficácia em distâncias curtas e médias (até quinze metros). Esta foi utilizada com sucesso na imobilização química à distância de taiassuídeos (caititus e queixadas) e suídeos-ferais (porco-monteiro) na Fundação RioZoo e no Pantanal Matogrossense. O dispositivo mostrou ser prático, permitindo ser recarregado de modo simples e garantindo um satisfatório grau de penetração no tegumento do animal independentemente de sua resistência, porém sem maiores riscos de perfuração de partes vitais ou fratura de ossos.
\end{abstract}

Descritores: armas anestésicas, manejo de fauna silvestre, zoológicos, manejo pecuário.

\begin{abstract}
A ranged intramuscular drug injection on zoo animals, cattle, and wildlife involves either the use of expensive equipments such as air-guns and firearms or improvised darts shoot by blow-guns with unsatisfactory results in regards to the range and the volume of the drug injected. Taking into account the economic reality of brazilian veterinary and zoological institutions, it was designed an arrow-like device, built with cheap, but lasting materials, to be used with commercial or self-made crossbows with greater results on short or medium ranges (up to fifteen meters long). This arrow has been successfully used on the ranged chemically induced immobilization of peccaries and feral-hogs at the zoo in Rio de Janeiro (Fundação RioZoo) and at the wetlands of Pantanal Matogrossense. This user-friendly device is easily reloaded; it shows good results regardless of the thickness of the animal skin, whereas it safeguards the animal from deadly piercing or broken bones.
\end{abstract}

Key words: ranged chemical restraint, wildlife-zoo-cattle management. 


\section{INTRODUÇÃO}

Existem dois métodos de inoculação de drogas à distância em animais via dardos: armas de ar comprimido e de fogo com câmaras de expansão de gases, ou por zarabatanas. Armas de fogo são caras, além dos custos de regulamentação destas às leis brasileiras [2]. Rifles de ar comprimido, dardos, cilindros de $\mathrm{CO}_{2}$ variam de US\$ 385-800 de acordo com a distância objetivada, segundo o catálogo da Cap-Chur ${ }^{\circledR}$ [1]. A importação pode duplicar tais valores. No Brasil, equipamentos para longas distâncias acabam sendo adaptados para distâncias curtas, após seguidas reduções da pressão do cilindro de $\mathrm{CO}_{2}$. Além do desgaste do material, a pressão não é reduzida satisfatoriamente, aumentando o risco de perfuração de áreas vitais pelo dardo ou fratura de ossos.

Zarabatanas disparam dardos fabricados com seringas descartáveis dotadas de dois êmbolos: um mediano móvel e um fixado posteriormente. A droga é colocada do êmbolo mediano até a abertura frontal, onde é posicionada a agulha com uma abertura lateral vedada por uma rolha de borracha. Através do êmbolo posterior, é injetado ar sob pressão. Ao atingir o alvo, a borracha de vedação da agulha é deslocada e o sistema se abre: a câmara posterior se expande, movimentando o êmbolo mediano e injetando a droga. A potência do sopro do usuário impõe limitações de distância e de volume do fármaco, além da eventual baixa penetração devido às características tegumentares do animal.

Objetivou-se disponibilizar uma tecnologia de baixo custo para inoculação de drogas a curtas e médias distâncias com baixo risco à integridade física do animal. Este dispositivo foi concebido sob a forma de uma flecha de besta, cujo custo no Brasil, oscila de US\$ 50-350.

\section{MATERIAIS E MÉTODOS}

O funcionamento desta flecha é semelhante ao descrito para o dardo de zarabatana, no entanto, sua construção envolve maior complexidade. O dispositivo foi desenvolvido e utilizado para imobilização e captura temporária de taiassuídeos e suídeos ferais no Pantanal da Nhecolândia na Fazenda Nhumirim da Empresa Brasileira de Pesquisa Agropecuária/Centro de Pesquisas Aplicadas do Pantanal (EMBRAPA - CPAP), Mato Grosso do Sul, para estudo de ecomorfologia comparativa e conservação das espécies nativas [4,5]. Ambos os grupos são dotados de uma pe- lagem espessa e tegumento resistente [4]. Foram realizados, previamente, testes de simulação em alvos artificiais e testado in vivo na Fundação RIOZOO, Prefeitura do Rio de Janeiro.

Os materiais utilizados na construção da flecha injetora seguem listados: tubo de alumínio de $1 / 2$ ' $(\sim 1,27 \mathrm{~cm})$ de $\varnothing$ externo, espessura de $2 \mathrm{~mm}$ e $28 \mathrm{~cm}$ de comprimento; folha de acetato colorida; seringa descartável de $5 \mathrm{ml}$, rolhas de borracha ou silicone; agulha de aço inoxidável 50x15 (não-descartáveis); arame metálico; e, por fim, um comprimido efervescente farmacêutico. Os utensílios usados na manufatura do dispositivo são: torno mecânico, furadeira elétrica ou manual ou micro-retífica, lixas d'água (grão 160 e 400), lima de metal, solda (ou resina epóxi), tesoura e cola-de-contato.

O tubo de alumínio foi torneado para ter seu diâmetro externo reduzido em $1 \mathrm{~mm}$. Uma das extremidades foi mantida no diâmetro original até a distância de $1,5 \mathrm{~cm}$. O tubo foi lixado seqüencialmente para que a superfície torneada ficasse o mais lisa possível. Na extremidade oposta, furou-se diametralmente o tubo à cerca de $3 \mathrm{~mm}$ da borda final. Rolhas de borracha (ou silicone) ajustadas ao diâmetro interno do tubo foram utilizadas como êmbolo intermediário e de fechamento da porção posterior do tubo (Figura 1).

A ponta da flecha foi confeccionada com a parte anterior de uma seringa descartável de $5 \mathrm{ml}$, cortada a 1,5 cm de distância da rosca. Esta peça foi fixada, após rápida dilatação por calor, na porção anterior do tubo (extremidade de maior diâmetro). A abertura apical da agulha foi selada com solda e uma nova abertura foi feita lateralmente à cerca de $1,5 \mathrm{~cm}$ da ponta. Uma pequena rolha de borracha foi utilizada para a vedação do orifício lateral da agulha. Utilizouse um tubo de alumínio de $1,5 \mathrm{~cm}$ como reforço da ponta plástica da flecha (Figura 2).

As aletas ou "penas" de estabilização da flecha podem ser em número de duas ou três, de acordo com a besta utilizada. Duas folhas de acetato foram coladas e recortadas em forma de meia pena com $9 \mathrm{~cm}$ de comprimento e 1,2 cm de largura da metade para o fim desta. Uma pequena borda dobrada foi feita para permitir a colagem desta na haste da flecha. $\mathrm{O}$ acetato colorido facilita a localização em caso de perda da flecha.

A montagem final do dispositivo é feita após a limpeza e esterilização dos componentes. O êmbolo mediano é inserido no corpo da flecha com o uso de 


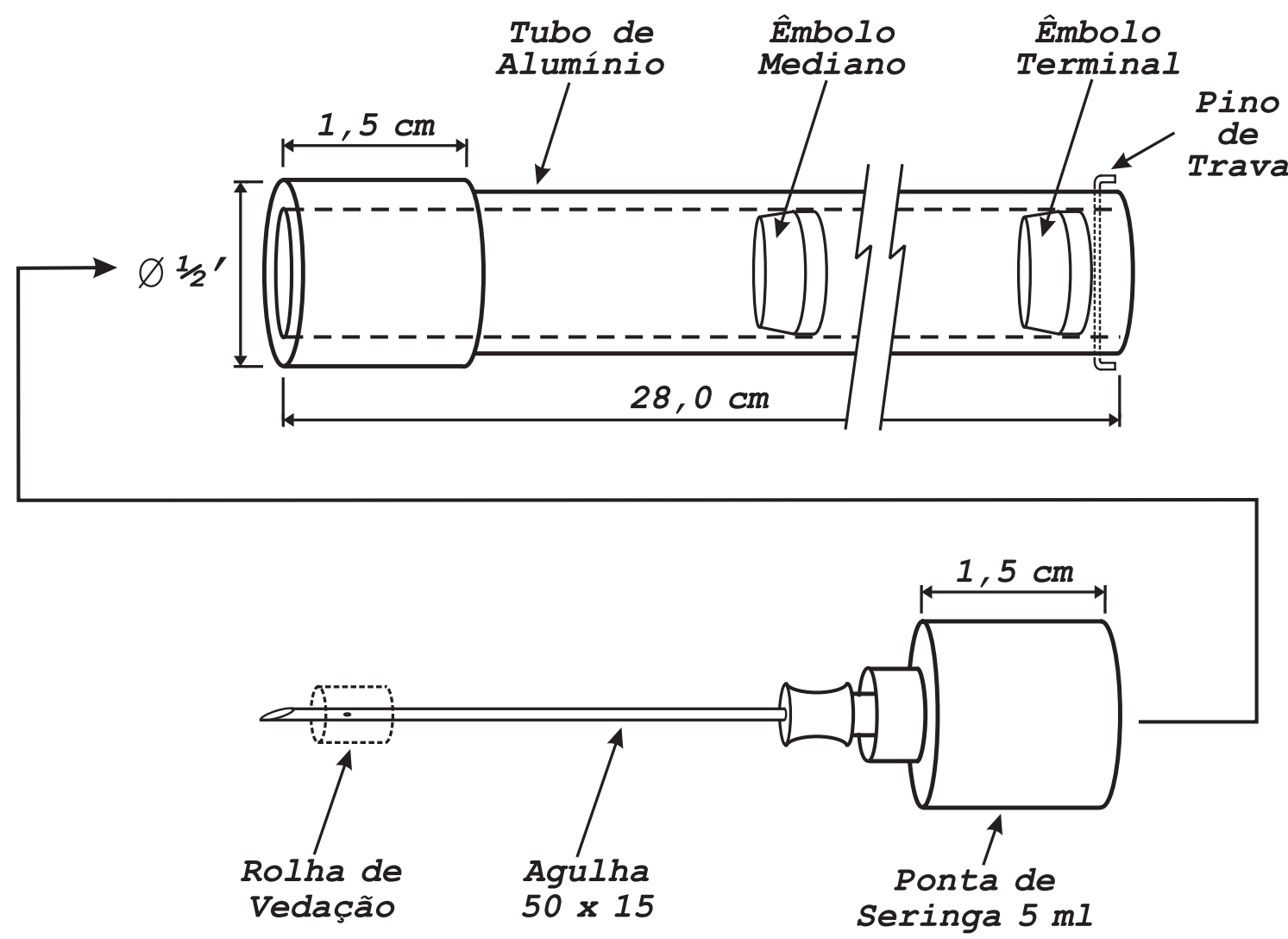

Figura 1. Esquema técnico do dispositivo injetor com dimensões e posicionamentos de componentes.

uma haste. A droga a ser administrada é previamente dosada e colocada na flecha através da abertura da seringa posicionada na ponta desta. A agulha é posicionada e o êmbolo mediano é empurrado com a haste até a retirada completa das bolhas de ar. Veda-se a abertura lateral da agulha com a pequena rolha de borracha isolando a chamada "câmara de injeção" - do êmbolo mediano até a ponta da agulha. Na abertura posterior da flecha é inserido meio comprimido efervescente de uso farmacêutico comum (é necessário que nesta secção da flecha seja eliminada toda a umidade) e em seguida a rolha de borracha terminal é posicionada fechando a abertura da "câmara de compressão". O pino-de-trava feito de arame é então fixado transversalmente através dos orifícios laterais abertos na porção posterior da flecha de modo a impedir a saída desta rolha terminal (Figura 1). Deste modo todo o sistema está hermético e pronto para ser transportado.

A preparação do disparo é feita com a aplicação de uma injeção de água através da rolha de borracha terminal de modo que a água entre em contato com a substância efervescente. Com a câmara de compressão repleta de $\mathrm{CO}_{2}$, a flecha é posicionada na besta para que o atirador busque o melhor posicionamento possível para o disparo. Este dispositivo permanecerá apto a ser disparado por várias horas. $\mathrm{O}$ próprio cuidado com o qual foi confeccionado garantirá o hermetismo do sistema.

A flecha ao ser disparada, atinge o alvo e a agulha penetra o tegumento chegando à musculatura do animal. O impacto provoca o recolhimento da vedação de borracha até a base da agulha abrindo o sis-

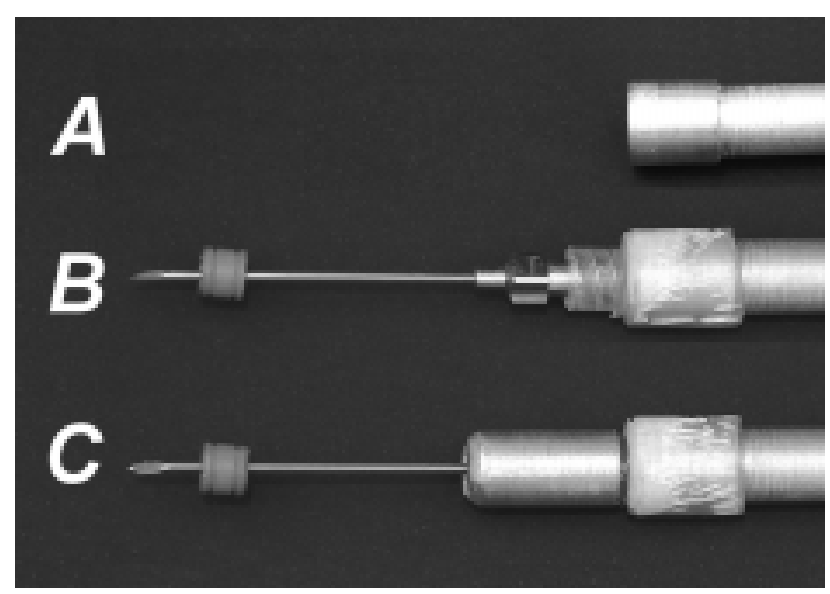

Figura 2. Detalhes da ponta do dispositivo injetor ao longo de sua montagem (A, B e C). O tubo de alumínio utilizado como reforço da ponta da flecha é observado em C. 
tema. A pressão causada pela liberação do $\mathrm{CO}_{2}$ na câmara de compressão desloca rapidamente o êmbolo mediano, promovendo a injeção da droga.

Este dispositivo foi usado através de uma besta artesanal com arco de aço e cerca de 70lb de potência (Figura 3). Devido à baixa potência da besta, alguns disparos tiveram que ser levemente balísticos (mediante inclinação), porém sem prejuízos relevantes à pontaria.

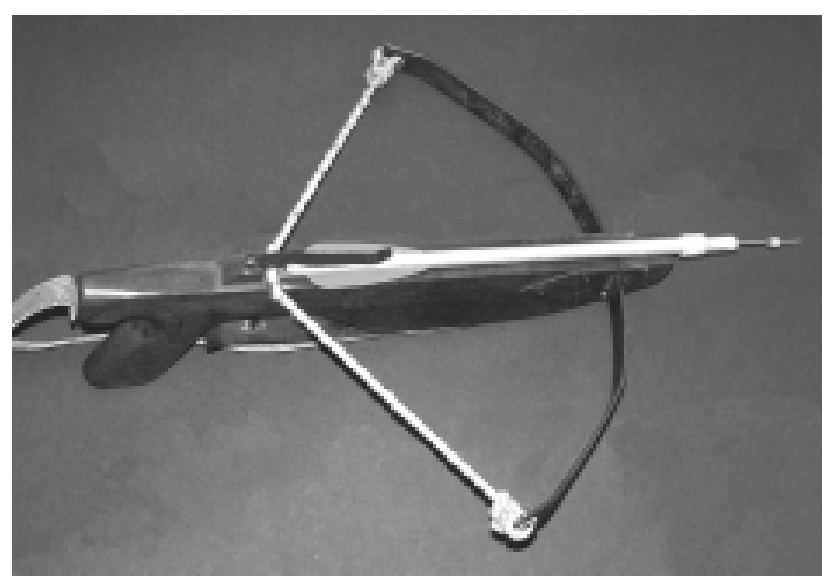

Figura 3. Dispositivo injetor montado em uma besta artesanal pronto para o disparo.

\section{RESULTADOS}

Em testes de liberação manual da borracha de vedação da agulha o dispositivo armado esvaziou o conteúdo da câmara de injeção de forma rápida e contínua em $100 \%$ das vezes (total $=10$ ). Em testes contra alvos artificiais, com uma carga máxima na câmara de injeção de $20 \mathrm{ml}$, foram obtidos disparos retos de até 4 metros e, com efeito balístico, até 9 metros. A redução do volume a ser injetado possibilitou um alcance com precisão até 15 metros. Em todos os disparos a câmara de injeção foi esvaziada completamente. No teste in vivo, o dispositivo foi utilizado para a anestesia, mediante associação de cloridrato de xilazina e cloridrato de cetamina, de um queixada (Tayassu pecari) sob a supervisão técnica dos funcionários da Fundação RioZoo, à curta distância (cerca de 5 metros) [4,6]. Posteriormente, este dispositivo foi utilizado com sucesso no campo para a captura de dois catetos ( $P e$ cari tajacu), um queixada (T. pecari) e dois porcosmonteiros (Sus scrofa - morfotipo feral) na Fazenda Nhumirim no Pantanal da Nhecolândia, com apoio técnico da EMBRAPA - CPAP [4]. As distâncias de disparo foram estimadas entre 7 e 10 metros de distância em ambiente natural. Os animais estudados receberam satisfatoriamente a dosagem da droga.

\section{CONCLUSÃO}

Os centros de pesquisa zoológica e veterinária no Brasil dispõem de recursos limitados. Os equipamentos comerciais utilizados para a administração de drogas injetáveis à distância são, neste sentido, pouco acessíveis. $\mathrm{O}$ dispositivo aqui descrito, devido à sua simplicidade de confecção e eficiência na administração de drogas injetáveis à curta e média distâncias, é proposto como uma alternativa viável para estas instituições. O uso do dispositivo para anestesia de animais em zoológico e no campo demonstrou sua funcionalidade. $\mathrm{O}$ uso desta flecha em bestas comerciais com maior desempenho de disparo pode maximizar as distâncias citadas, aumentando ainda mais a versatilidade deste dispositivo.

\section{REFERÊNCIAS}

1 Cap-Chur ${ }^{\circledR}$. 2006. Palmer Cap-Chur Price List. Disponível em: <http://www.palmercap-chur.com/products.html>. Acessado em 02/10/2006.

2 Hossepian Jr. A. 2000. Instrumentos para anestesia à distância são fundamentais para a segurança na saúde pública. Clínica Veterinária. 27: 54-54.

3 Ko J.C., Williams B.L., Smith V.L., McGrath C.J. \& Jacobson J.D. 1993. Comparison of Telazol ${ }^{\circledR}$, Telazol $^{\circledR}-\mathrm{ketamine}$, Telazol $^{\circledR}$-xylazine, and Telazol ${ }^{\circledR}$-ketamine-xylazine as chemical restraint and anesthetic induction combination in swine. Laboratory Animal Science (Chicago). 43: 476-480.

4 Sicuro F.L. 1996. Inferências acerca da coexistência de taiassuídeos e suídeos ferais (Mammalia, Artiodactyla), no Pantanal da Nhecolândia (MS): um modelo ecomorfológico. 162f. Rio de Janeiro, RJ Dissertação (Mestrado em Ciências Biológicas - Zoologia) - Programa de Pós-graduação em Ciências Biológicas, Museu Nacional, Universidade Federal do Rio de Janeiro.

5 Sicuro F.L. \& Oliveira L.F.B. 2002. Coexistence of peccaries and feral hogs in brazilian Pantanal wetland: an ecomorphological view. Journal of Mammalogy 83: 207-217.

6 Sweitzer R.A., Ghnelm G.S., Gardner I.A., Van Vuren D., Gonzales B.J. \& Boyce W.M. 1997. Immobilization and physiological parameters associated with chemical restraint of wild pigs with Telazol ${ }^{\circledR}$ and xylazine hydrochloride. Journal of Wildlife Diseases. 33: 198-205.

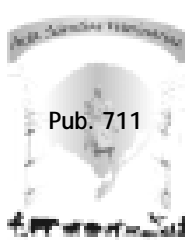

